\title{
High Frequency Alfvén Eigen Modes Observed in LHD
}

\author{
Hiroyuki HIGAKI ${ }^{\text {a)}}$, Makoto ICHIMURA, Daisuke INOUE, Ken-ichiro NAKAGOME, \\ Yuusuke YAMAGUCHI, Shingo KAKIMOTO, \\ Ryuhei KUMAZAWA ${ }^{1)}$, Tetsuo WATARI ${ }^{1)}$, Takashi MUTOH ${ }^{1)}$, Tetsuo SEKI ${ }^{1)}$ and Kenji SAITO ${ }^{1)}$ \\ Plasma Research Center, University of Tsukuba, Tsukuba 305-8577, Japan \\ ${ }^{1)}$ National Institute for Fusion Science, Toki 509-5292, Japan
}

(Received 26 December 2005 / Accepted 12 May 2006)

\begin{abstract}
Alfvén eigen modes were observed in the Large Helical Device for various magnetic field strengths and plasma densities. It was found that Alfvén eigen modes could be excited at much higher frequencies $(0.5 \sim$ $2.5 \mathrm{MHz})$ and higher magnetic fields $(1.5 \sim 2.83 \mathrm{~T})$ than previously reported. A part of the estimated Alfvén velocities $\left(2 \sim 6 \times 10^{7} \mathrm{~m} / \mathrm{s}\right)$ exceeded the thermal velocity of injected neutral beams $\left(\sim 4.1 \times 10^{6} \mathrm{~m} / \mathrm{s}\right)$.
\end{abstract}

(C) 2006 The Japan Society of Plasma Science and Nuclear Fusion Research

Keywords: Alfvén eigen mode, Alfvén velocity, neutral beam injection, Large Helical Device

DOI: $10.1585 /$ pfr. 1.034

When high energy ions are confined in a magnetized plasma, various fluctuations are excited. For example, ion cyclotron emission is observed in Tokamaks associated with the injection of high energy neutral beams (NB) [1] and Alfvén eigen modes (AEs) are also observed with NB injection in many fusion-oriented devices including the LHD [2,3]. High energy ions produced by ion cyclotron heating $(\mathrm{ICH})$ can also excite these fluctuations. Investigating these fluctuations is important because they degrade the fusion reaction rate and neutron yield.

In the present experiment, magnetic probes (MP) were employed to observe these fluctuations excited by NB and/or high power ICH injection in the LHD. Signals from MPs were recorded by a sampling oscilloscope (250 Ms/s). So far, fluctuations were observed in NB-sustained plasmas with field strength $B$ on a magnetic axis ranging from 1.0 to $2.8 \mathrm{~T}$, when the plasma density is lower than $\sim 10^{19} \mathrm{~m}^{-3}$. An example is shown in Fig. 1 for $B=2.75 \mathrm{~T}$. Shown in Fig. 1 (a) is the plasma density $n_{\mathrm{e}}$ near the magnetic axis, which was estimated by the measured line density. In Fig. 1 (b), the contour plot of the fast Fourier transformed (FFT) power spectrum obtained from a MP signal is shown with the frequency resolution $\Delta f \sim 15 \mathrm{kHz}$. The darker tone indicates the higher power. A FFT power spectrum is plotted in Fig. 1 (c) at $0.92 \mathrm{sec}$. The observed frequency can be fitted well with the $n_{\mathrm{e}}^{-1 / 2}$ fitting curve as shown in Fig. 1 (d).

Accumulating these fluctuation signals at various $B$ and $n_{\mathrm{e}}$, it was found that the frequencies of observed fluctuations were proportional to $B$ and $n_{\mathrm{e}}^{-1 / 2}$ as shown in Fig. 2 (a). Dashed lines are $n_{\mathrm{e}}^{-1 / 2}$ fitting curves for the data. For the sake of clarity, observed frequencies are nor-

author'se-mail: hhigaki@hiroshima-u.ac.jp

a) Present address: Graduate School of Advanced Sciences of Matter, Hiroshima University, Higashi-Hiroshima 739-8530, Japan
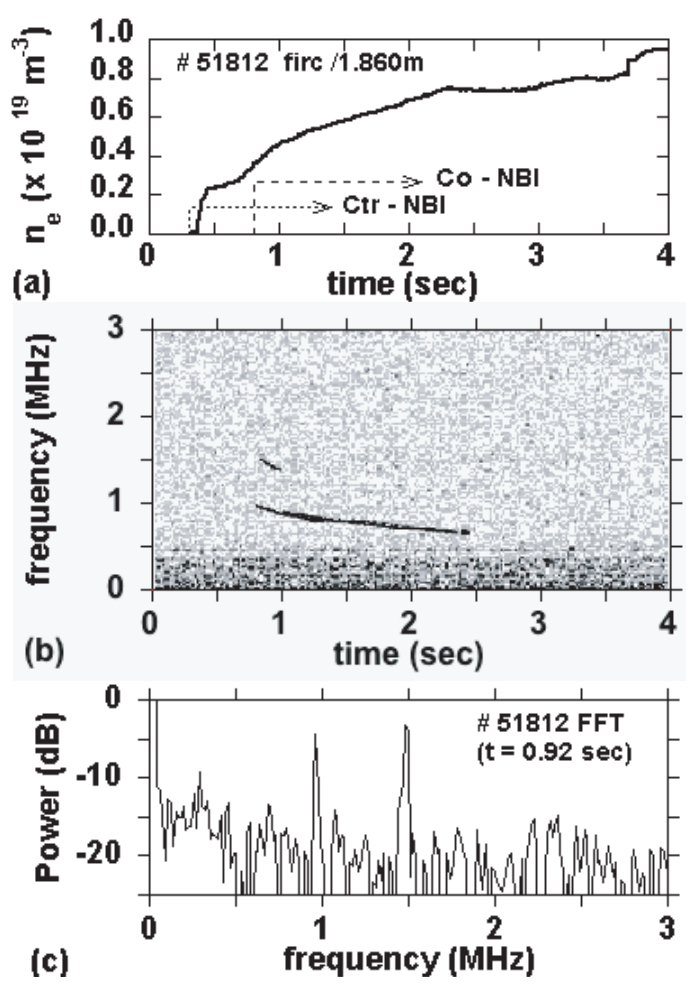

(c)

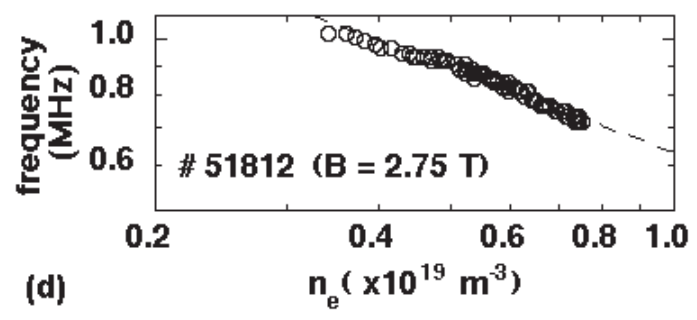

Fig. 1 (a) Plasma density near the magnetic axis. (b) Time evolution of FFT power spectrum. (c) Example of FFT power spectrum at $0.92 \mathrm{sec}$. (d) The dependence of observed frequency on $n_{\mathrm{e}}$. The frequency is found to be proportional to $n_{\mathrm{e}}^{-1 / 2}$. 


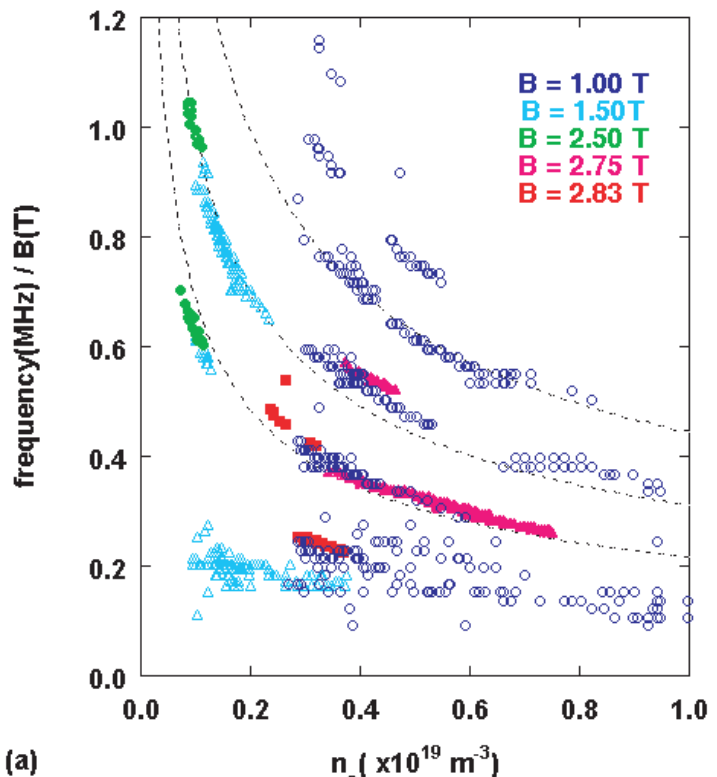

(a)
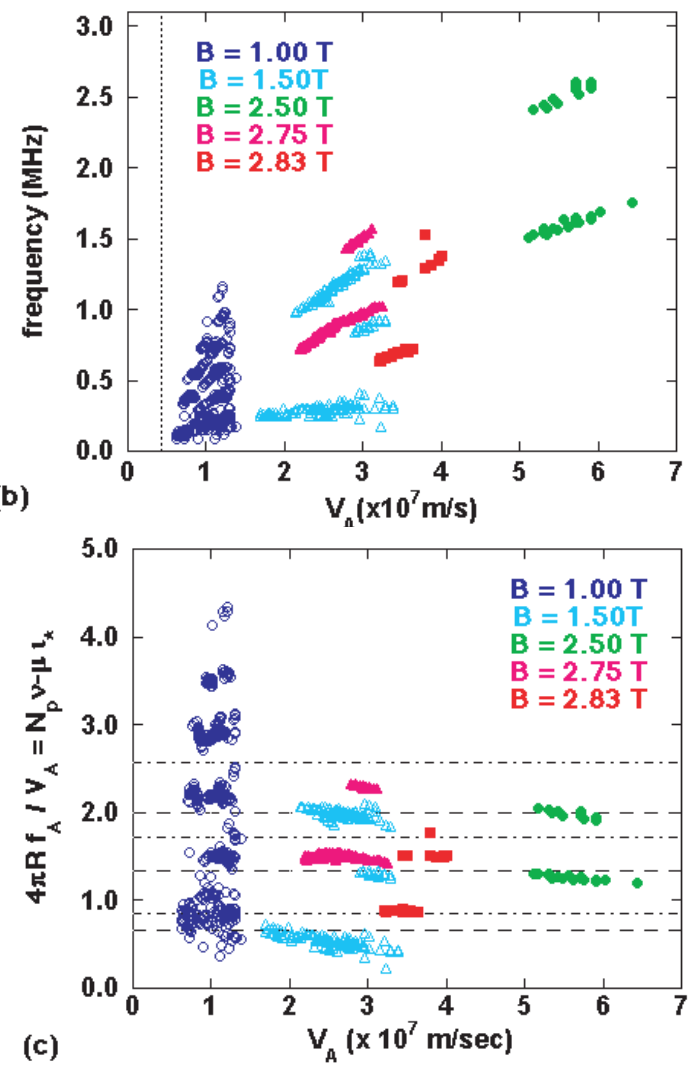

Fig. 2 (a) Normalized AE frequency as a function of plasma density. (b) Observed AE frequency as a function of estimated $V_{\mathrm{A}}$. (c) Effective wave numbers can be obtained as a function of $V_{\mathrm{A}}$. The dashed lines correspond to both TAE and EAE and the dashed dotted lines correspond to NAE. malized by $B$. The dependencies on $B$ and $n_{\mathrm{e}}$ imply that the fluctuations are Alfvén waves whose phase velocity is given by $V_{\mathrm{A}}=2 \pi f_{\mathrm{A}} / k=B / \sqrt{\mu_{0} n_{\mathrm{e}} m}$. Since the fluctuations are discretely excited, it is suggested that these fluctuations are AEs. Here, $k$ is the wave number which was not measured in the present experiment, $\mu_{0}$ is the permeability, and $m$ denotes the effective ion mass. As far as AEs in the LHD are concerned, it was found that AEs were excited at much higher frequencies and higher magnetic fields than reported previously [2,3].

In Fig. 2 (b), the observed AE frequency was plotted as a function of estimated $V_{\mathrm{A}}$, assuming that ions were all protons. Although $V_{\mathrm{A}}$ depends on the local plasma density and magnetic field, the estimation with $n_{\mathrm{e}}$ and $B$ is feasible with an error of $40 \%$. However, $V_{\mathrm{A}}$ is strongly affected by heavy ion impurities. If $8 \% \mathrm{Ar}^{+}$ions are included, the estimated $V_{\mathrm{A}}$ becomes half and $100 \% \mathrm{Ar}^{+}$ions makes $V_{\mathrm{A}}$ about one-sixth. Thus, impurities and density profiles must be considered properly for detailed analysis. The vertical dotted lines in Fig. 2 (b) and (c) denote the velocity $v_{\mathrm{b}} \sim$ $4.1 \times 10^{6} \mathrm{~m} / \mathrm{sec}$ of $180 \mathrm{keV}$ protons supplied through NB injection into the plasma. It is seen that the phase velocities of some data points are larger than $v_{\mathrm{b}}$ even if the effect of impurities is considered.

Although it should be investigated carefully, the excited modes can be roughly estimated by

$$
\frac{4 \pi R f_{\mathrm{A}}}{V_{\mathrm{A}}}=N_{\mathrm{p}} v-\mu \iota_{*}, \quad\left(\iota_{*}=\frac{2 n+v N_{\mathrm{p}}}{2 m+\mu}\right)
$$

with the plasma major radius $R$ and the number of toroidal field period $N_{\mathrm{p}}=10$, which are the same notations given in Refs. [2,3]. The obtained numbers plotted in Fig. 2 (c) suggest that toroidicity-induced AE (TAE) with $\mu=1$, $v=0, m=1, n=1,2,3$, ellipticity-induced AE (EAE) with $\mu=2, v=0, m=2, n=1,2,3$, and noncircular triangularity-induced AE (NAE) with $\mu=3, v=0, m=2$, $n=1,2,3$ are candidates for the observed AEs.

Since AEs affect the behavior of high energy ions in fusion plasmas, the modes of the excited fluctuations should be identified with an array of MPs in future experiments.

The author H.H. acknowledges his discussion with Dr. S. Yamamoto. This work is supported by NIFS03KLRR001 and NIFS04KUGM007.

[1] G.A. Cottrell and R.O. Dendy, Phys. Rev. Lett. 60, 33 (1988).

[2] S. Yamamoto, K. Toi, N. Nakajima et. al., Phys. Rev. Lett. 91, 245001 (2003).

[3] S. Yamamoto, K. Toi, S. Ohdachi et. al., Nucl. Fusion 45, 326 (2005). 